\title{
Discovery of a second pulsating intermediate helium-enriched sdOB star ${ }^{\star}$
}

\author{
M. Latour ${ }^{1}$, E. M. Green ${ }^{2}$, and G. Fontaine ${ }^{3}$ \\ ${ }^{1}$ Institute for Astrophysics, Georg-August-University, Friedrich-Hund-Platz 1, 37077 Göttingen, Germany \\ e-mail: marilyn.latour@uni-goettingen.de \\ 2 Steward Observatory, University of Arizona, 933 North Cherry Avenue, Tucson, AZ 85721, USA \\ e-mail: bgreen@as. arizona.edu \\ 3 Département de Physique, Université de Montréal, Succ. Centre-Ville, 6128 Montréal, QC H3C 3J7, Canada \\ e-mail: fontaine@astro.umontreal.ca
}

Received 19 February 2019 / Accepted 8 March 2019

\begin{abstract}
We present the discovery of long-period, low-amplitude, $g$-mode pulsations in the intermediate He-rich hot subdwarf (sdOB) star Feige 46. So far, only one other He-enriched sdOB star (LS IV-14 $\left.{ }^{\circ} 116\right)$ was known to exhibit such pulsations. From our groundbased light curves of Feige 46, we extracted five independent periodicities ranging from 2294 s to $3400 \mathrm{~s}$. We fit our optical spectrum of the star with our grid of non-local thermodynamic equilibrium (NLTE) model atmospheres and derived the following atmospheric parameters: $T_{\text {eff }}=36120 \pm 230 \mathrm{~K}, \log g=5.93 \pm 0.04$, and $\log N(\mathrm{He}) / N(\mathrm{H})=-0.32 \pm 0.03$ (formal fitting errors only). These parameters are very similar to those of LS IV $-14^{\circ} 116$ and place Feige 46 well outside of the instability strip where the hydrogen-rich $g$-mode sdB pulsators are found. We used the Gaia parallax and proper motion of Feige 46 to perform a kinematic analysis of this star and found that it likely belongs to the Galactic halo population. This is most certainly an intriguing and interesting result given that LS IV $-14^{\circ} 116$ is also a halo object. The mechanism responsible for the pulsations in these two peculiar objects remains unclear, but a possible scenario involves the $\epsilon$-mechanism. Although they are the only two members in their class of variable stars, these pulsators appear to have more in common than just their pulsation properties.
\end{abstract}

Key words. subdwarfs - stars: oscillations - stars: individual: Feige 46

\section{Introduction}

The vast majority of subdwarf $\mathrm{B}(\mathrm{sdB}) \operatorname{stars}^{1}$ are compact, hot, helium-core burning objects that lost almost all of their hydrogen envelope before the He-flash, which left them unable to sustain H-shell burning. Consequently, their masses are close to the canonical value required for the He-flash $\left(\sim 0.48 M_{\odot}\right)$, to which their remaining hydrogen envelope contributes only very little $\left(\lesssim 0.02 M_{\odot}\right)$. The discovery of the first pulsating $\mathrm{sdB}$ stars (Kilkenny et al. 1997) two decades ago proved to be a stepping stone for our understanding of these particular objects (see Heber 2016 and Charpinet et al. 2016 for recent reviews). Asteroseismic modeling of these objects has allowed probing their structural properties, such as core size and composition (e.g., Charpinet et al. 2011; Van Grootel et al. 2013), rotation (Charpinet et al. 2018), and stellar masses (Fontaine et al. 2012 and references therein). Pulsating hot subdwarfs show pressure $(p-)$ and gravity $(g-)$ mode instabilities. The former appear as rapid periodic variations of a few millimagnitudes on a timescale of a few minutes, while the latter are characterized

\footnotetext{
* The reduced spectrum is only available at the CDS via anonymous ftp to cdsarc.u-strasbg.fr (130.79.128.5) or via http: //cdsarc.u-strasbg.fr/viz-bin/qcat?J/A+A/623/L12

1 We note that the term "sdB stars" is often understood to include the slightly hotter sdOB stars because they are nearly identical in an evolutionary sense.
}

by slower variations with periods between 0.5 and $4 \mathrm{~h}$ and even smaller amplitudes, $\lesssim 1$ millimag. The two types of sdB pulsation modes are excited in two distinct instability regions in the $\log g-T_{\text {eff }}$ plane, with the slow, $g$-mode pulsators (formally referred to as V1093 Her stars) found at cooler effective temperatures $(22-29 \mathrm{kK})$ than their rapidly pulsating V361 Hya counterparts $(29-36 \mathrm{kK})$. Interestingly, some stars at the boundary between the two instability domains show both $p$ - and $g$-mode pulsations (e.g., Schuh et al. 2006). The instability regions are observationally and theoretically well defined (Charpinet et al. 2001; Bloemen et al. 2014), although the boundary between the two types becomes more fuzzy when viewed with space-borne sensitivity. Both classes of pulsating hot subdwarfs can be successfully modeled in terms of the same driving mechanism: a classical opacity $(\kappa)$ mechanism associated with a local overabundance of iron and nickel in the driving region (Charpinet et al. 1997).

While the majority of pulsating hot subdwarfs belong to these two classes, each well described by asteroseismology, a few of these pulsators remain theoretically challenging: the few hotter sdO pulsators (Randall et al. 2016; Kilkenny et al. 2017), and the very unusual sdOB star LS IV-14 ${ }^{\circ} 116$ (Green et al. 2011). Although the latter has been previously referred to as an sdB star, its spectrum, showing a strong He II $4686 \AA$ line, is better classified as $\mathrm{sdOB}$, a more specific term for objects in the transition region between $\mathrm{sdB}$ and sdO stars. LS IV $-14^{\circ} 116$ 

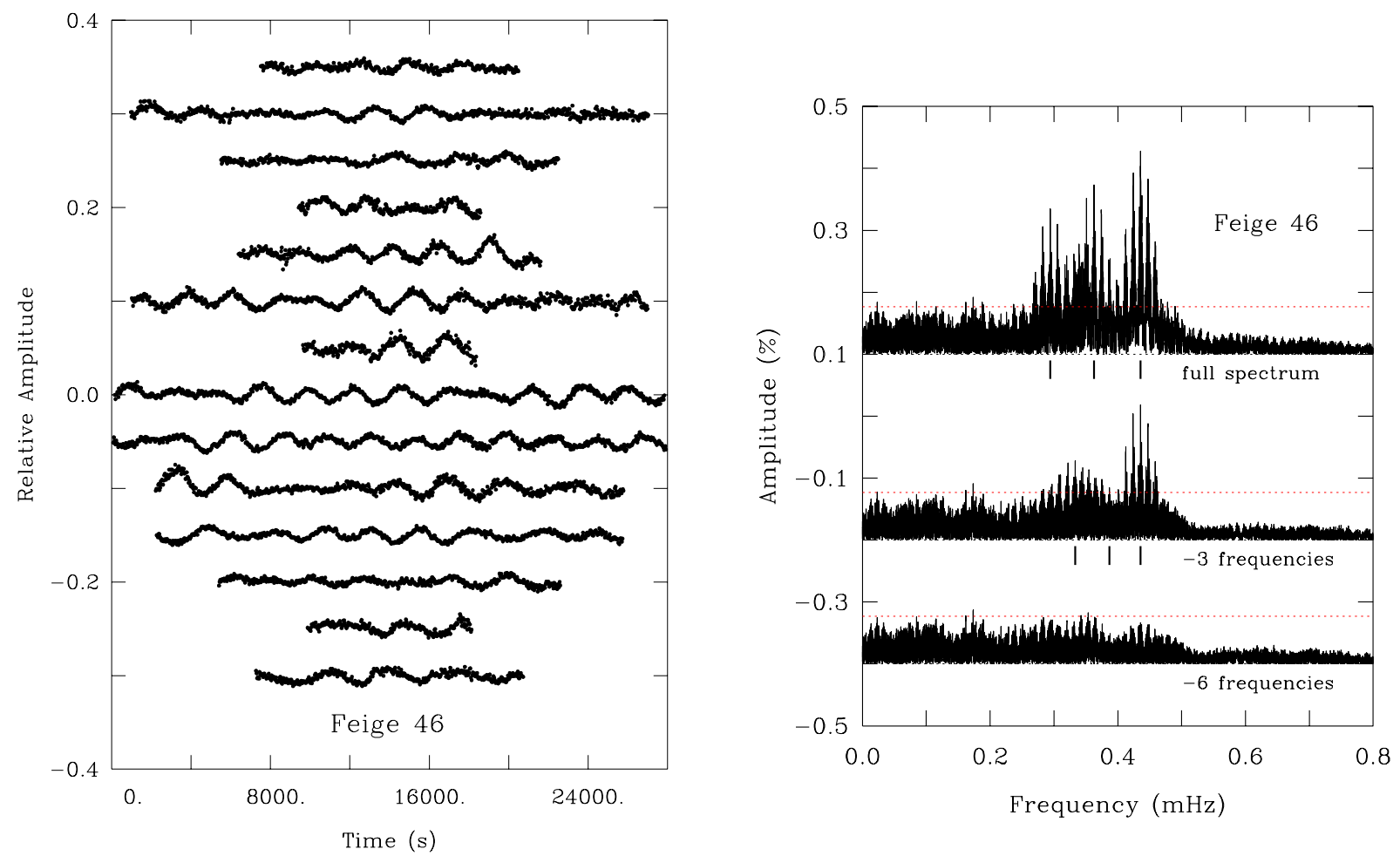

Fig. 1. Left panel: all light curves obtained for Feige 46. The data have been shifted arbitrarily along the $x$ - and $y$-axes for visualization purposes. The $y$-axis is expressed in units of fractional brightness intensity (the residual amplitude of the pulsation relative to the mean brightness of the star). Right panel: zoomed-in view of the Fourier transform of the entire data set in the $0-0.8 \mathrm{mHz}$ range where the periodicities are found. The lower transforms show the successive steps of prewhitening by the three and six frequencies indicated. The dashed horizontal lines indicate the $4 \sigma$ noise level.

shows long-period ( $P \sim 2000-5000$ s), multiperiodic luminosity variations even though its atmospheric parameters place the star on the hotter side of the $p$-mode instability strip, where its long pulsation periods cannot be explained by the iron-bump $\kappa^{-}$ mechanism (Ahmad \& Jeffery 2005; Green et al. 2011). In addition to its distinctive pulsation properties, LS IV $-14^{\circ} 116$ has very peculiar atmospheric abundances: it contains the highest overabundances of Sr, Y, and $\mathrm{Zr}$ that have been reported in a hot subdwarf, and it is also one of the very few subdwarfs known to have an atmosphere that is enriched in helium (Naslim et al. 2011). In addition, Randall et al. (2015) found that it belongs to the halo population based on its kinematic properties. The star was therefore suggested to be the prototype of a new class of pulsating hot subdwarfs referred to as He-sdBV (Kilkenny et al. 2010). However, LS IV $-14^{\circ} 116$ remained the only member of its class for almost a decade. We note that two other variable helium-enriched hot subdwarfs were discovered with the Kepler satellite (UVO 0825+15 and KIC 1718290; Jeffery et al. 2017; Østensen et al. 2012), but because of their discrepant pulsation properties and, in the case of KIC 1718290, lower $T_{\text {eff }}$, they probably do not belong to the same class of pulsators as LS IV $-14^{\circ} 116$.

In this Letter, we present Feige 46 as the long sought-after second member of the He-sdBV class. Our extensive photometric monitoring of the star has revealed multiperiodic variations that are very similar to those seen in LS IV $-14^{\circ} 116$, and our spectral analysis demonstrates that the atmospheric parameters of the two stars are strikingly similar. This discovery is all the more exciting because Feige 46 is bright enough $(V=13.2)$ to be observed by the TESS satellite and to allow high-resolution spectroscopic follow-up and more detailed investigation of its atmospheric properties.

\section{Pulsational properties}

The variable nature of Feige 46 was discovered in February 2018 during a photometric search for asteroseismically interesting hot subdwarf stars at the $1.55 \mathrm{~m}$ Kuiper telescope of Steward Observatory on Mt. Bigelow, using the Mont4K CCD camera $^{2}$. Following the realization that this star closely resembles LS IV $-14^{\circ} 116$, with low-amplitude luminosity variations on a timescale of about $45 \mathrm{~min}$ that are at odds with its sdOB spectrum (see Sect. 3), extensive follow-up was performed during 13 additional nights on the Kuiper telescope (see Table A.1). This resulted in $71.19 \mathrm{~h}$ of time-series observations with an average sampling time of $30 \mathrm{~s}$, taken through a broadband Schott 8612 filter. The images were reduced using standard IRAF photometric data reduction tasks. The light curves were constructed relative to five reasonably bright reference stars distributed symmetrically around Feige 46 in the $9.7^{\prime} \times 9.7^{\prime}$ Mont $4 \mathrm{k}$ field of view. The photometric aperture size was set to either 2.25 times the average FWHM of the six measured stars or 15 pixels $\left(=6.45^{\prime \prime}\right)$, whichever was smaller, to avoid contamination from the fainter visual companion 13.3" northwest of Feige 46. Light curves for each of the reference stars relative to the four others confirmed that none were variable above the level of the photometric noise. The light curves for Feige 46 are shown in the left panel of Fig. 1 in the same order as the observations in Table A.1.

The time-series photometry gathered for Feige 46 was analyzed in a standard way using a combination of Fourier analysis, least-square fits to the light curve, and prewhitening techniques as described in Billères et al. (2000). The light-curve analysis

\footnotetext{
2 http://james.as.arizona.edu/ psmith/61inch/
} instruments.html 
Table 1. Harmonic oscillations detected in the light curve of Feige 46.

\begin{tabular}{lcccc}
\hline \hline $\begin{array}{l}\text { Period } \\
(\mathrm{s})\end{array}$ & $\begin{array}{c}\text { Frequency } \\
(\mu \mathrm{Hz})\end{array}$ & $\begin{array}{c}\text { Amplitude } \\
(\%)\end{array}$ & $\begin{array}{c}\text { Phase } \\
(\mathrm{s})\end{array}$ & $(S / N)$ \\
\hline $2294.673 \pm 0.020^{a}$ & $435.792 \pm 0.004$ & $0.290 \pm 0.015$ & $420 \pm 19$ & 15.1 \\
$2296.064 \pm 0.024^{a}$ & $435.528 \pm 0.005$ & $0.243 \pm 0.015$ & $1056 \pm 23$ & 12.7 \\
$2585.797 \pm 0.083$ & $386.728 \pm 0.012$ & $0.090 \pm 0.015$ & $670 \pm 70$ & 4.7 \\
$2757.738 \pm 0.032$ & $362.616 \pm 0.004$ & $0.267 \pm 0.015$ & $2210 \pm 25$ & 13.9 \\
$2999.184 \pm 0.072$ & $333.424 \pm 0.008$ & $0.140 \pm 0.015$ & $1528 \pm 53$ & 7.3 \\
$3400.713 \pm 0.057$ & $294.056 \pm 0.005$ & $0.225 \pm 0.015$ & $2520 \pm 37$ & 11.7 \\
\hline
\end{tabular}

Notes. ${ }^{(a)}$ These modes are part of a possible rotation triplet.

revealed six frequency peaks, whose characteristics are listed in Table 1 . The phases given in the table are relative to the beginning of the first run on UT 2018 February 26. The uncertainties and the signal-to-noise ratio $(\mathrm{S} / \mathrm{N})$ were computed as described in Green et al. (2011). We found that the oscillation periods of Feige 46 are in the range of 2294-3400s, which overlaps the range of periods detected in LS IV $-14^{\circ} 116$ (1953-5083 s; Green et al. 2011) quite well. The low amplitudes are typical of $g$-mode pulsations. The right panel of Fig. 1 presents the Fourier amplitude spectrum of the full data set (upper curve) in the $0-0.8 \mathrm{mHz}$ range where the pulsating periods of Feige 46 are found. The lower curves are the resulting Fourier transforms after prewhitening by the indicated three and six frequency peaks. The structure of the main oscillation peak is compatible with the rotational splitting of an $l=1$ mode, of which two out of the three components have an $\mathrm{S} / \mathrm{N}$ above the $4.5 \sigma$ limit. A central component is present at a frequency of $435.670 \pm 0.014$ $\mu \mathrm{Hz}$, but only with an $S / N=3.4$. The average frequency difference $(\Delta f)$ between the peaks is $0.132 \mu \mathrm{Hz}$. Assuming that these are high-radial order $k$-modes, this frequency splitting indicates a slow rotation with a period of $\sim 43$ days.

\section{Spectroscopic properties}

Feige 46 is part of the Arizona-Montréal spectroscopic sample (Fontaine et al. 2014) and was observed multiple times with the $2.3 \mathrm{~m}$ Bok telescope at Steward Observatory on Kitt Peak between 2004 and 2011. The observations were obtained with the $\mathrm{B} \& \mathrm{C}$ spectrograph using the $400 \mathrm{~mm}^{-1}$ grating in first order and a $2.5^{\prime \prime}$ slit, resulting in a wide wavelength coverage $(3620-6900 \AA)$ and a rather low resolution of $8.7 \AA$. Our final spectrum with high $\mathrm{S} / \mathrm{N}$ was constructed from five medianfiltered individual spectra that were first cross-correlated to correct for possible variations in radial velocity (RV). However, no $\mathrm{RV}$ variations were found above the observational noise level $\left(\sigma \sim 10 \mathrm{~km} \mathrm{~s}^{-1}\right)$.

We derived the atmospheric parameters $\left(T_{\text {eff }}, \log g\right.$, and helium abundance) of Feige 46 using our grid of non-local thermodynamic equilibrium (NLTE) model atmospheres, which are especially well suited for analyses of sdB stars. The models and synthetic spectra were computed using the TLUSTY and SYNSPEC codes (Hubeny \& Lanz 2011), including a metallic composition typical of sdB stars (Blanchette et al. 2008; Brassard et al. 2010). Figure 2 shows the resulting best fit to the normalized Balmer and helium lines in the observed spectrum. We find $T_{\text {eff }}=36100 \pm 230 \mathrm{~K}, \log g=5.93 \pm 0.04$, and a helium abundance of $\log N(\mathrm{He}) / N(\mathrm{H})=-0.32 \pm 0.03$. The quoted uncertainties are formal errors returned by the $\chi^{2}$ minimization procedure and are therefore only lower limits.
Because the same model atmosphere grid was used and the spectra were obtained with an identical instrumental setup, the atmospheric parameters of $\mathrm{v}$ are directly comparable to those derived for LS IV $-14^{\circ} 116$ by Green et al. (2011), $T_{\text {eff }}=34900 \mathrm{~K}$, $\log g=5.9$ and $\log N(\mathrm{He}) / N(\mathrm{H})=-0.6$. The analysis of a highresolution spectrum of $\mathrm{LSIV}-14^{\circ} 116$ with the same models corroborates these values (Randall et al. 2015). Thus both stars have similar atmospheric parameters, in addition to comparable luminosity variations. Although Feige 46 is somewhat more helium-enriched than LS IV-14 116 , it is still classified as an intermediate He-rich subdwarf because its helium abundance is above solar $(\log N(\mathrm{He}) / N(\mathrm{H})=-1)$, but its atmosphere is still dominated, although only mildly, by hydrogen. The higher effective temperature and stronger He II 4686 line of Feige 46 with respect to LS IV $-14^{\circ} 116$ probably explains why Feige 46 was previously classified as an sdO star (Feige 1958; Graham 1970), while LS IV $-14^{\circ} 116$ was considered an sdB. The discrepancy in spectral type between the two stars is at odds with their similar atmospheric parameters. The two stars are more accurately described as sdOB because the $\mathrm{He}$ I and $\mathrm{He}$ I lines are clearly visible in their spectra. Therefore, we now refer to the two pulsators as He-sdOBV instead of using the previous He-sdBV nomenclature. This allows us to distinguish them from the cooler HesdBs (such as JL 87 and KIC 1718290) and the hotter He-sdOs, whose properties are different from those of LS IV $-14^{\circ} 116$ and Feige 46 (see also Sect. 2.2 of Heber 2009 for additional details on spectral classification). On the other hand, given the variety of spectral classification schemes and the growing number of pulsation properties that are discovered in hot subdwarfs, it might be clearer to refer to these two particular pulsators and potential future members of their class by the name of the prototype: LS IV-14 116 , also named V366 Aqr in the general catalog of variable stars (Samus et al. 2017).

Feige 46 has been little studied in the past. The most recent atmospheric analysis we found in the literature is that of Bauer \& Husfeld (1995), who derived $T_{\text {eff }}=37500 \pm 1500 \mathrm{~K}$, $\log g=5.25 \pm 0.25$, and abundances of $\mathrm{C}$ and $\mathrm{N}$ close to solar. They also estimated a spectroscopic distance of $1200_{-370}^{+535} \mathrm{pc}$. Their analysis was based on data obtained with the CASPEC Cassegrain echelle spectrograph mounted on the $3.6 \mathrm{~m}$ ESO telescope at La Silla Observatory in Chile. On the other hand, Kudritzki (1976) derived $T_{\text {eff }} \sim 41000 \mathrm{~K}$ and $\log g \sim 6.3$ based on Strömgren and Balmer jump colors.

Using newly available parallax and photometric measurements from Gaia, the derived atmospheric parameters can be verified by computing the spectroscopic distance and comparing this with the distance derived from the parallax. We performed this exercise for both LS IV-14 ${ }^{\circ} 116$ and Feige 46, assuming a mass of $0.47 M_{\odot}$, which is consistent with the mean $\mathrm{sdB}$ mass reported by Fontaine et al. (2012), and we used our model 


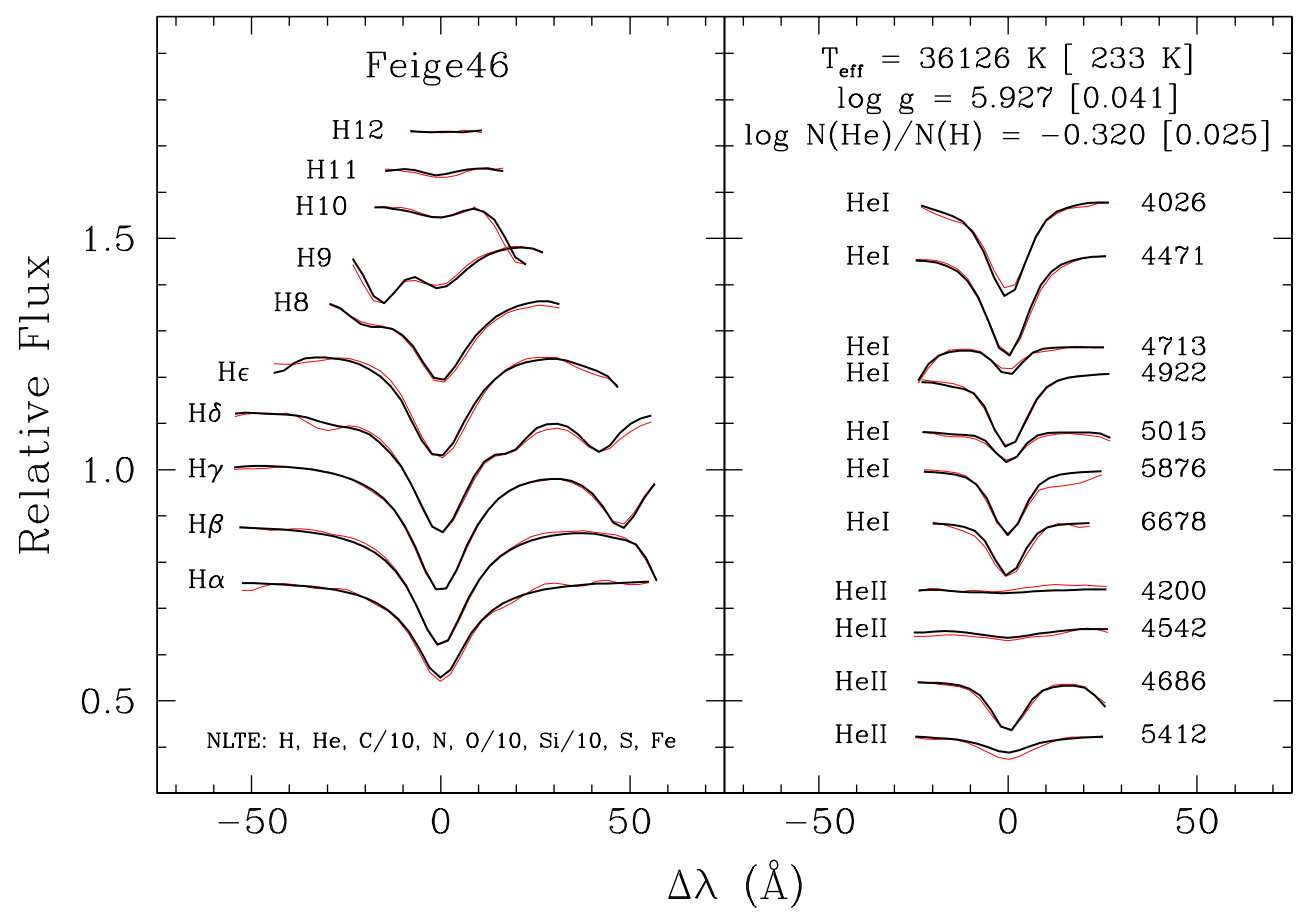

Fig. 2. Best fit (black curve) to the Balmer and helium lines visible in our low-resolution spectrum with high $\mathrm{S} / \mathrm{N}$ (red curve) of Feige 46. We used a three-dimensional grid $\left(T_{\text {eff }}, \log g\right.$, $N(\mathrm{He}) / N(\mathrm{H}))$ of NLTE model atmospheres, including a fixed metallic composition (solar $\mathrm{N}, \mathrm{S}$, and $\mathrm{Fe}, 1 / 10$ solar $\mathrm{C}, \mathrm{O}$, and $\mathrm{Si}$ ) that is typical for $\mathrm{sdB}$ stars. spectra to compute synthetic G(BP) and G(RP) absolute magnitudes. The comparison with the apparent magnitudes from Gaia DR2 resulted in spectroscopic distances of $426 \pm 27$ pc for LS IV $-14^{\circ} 116$ and $514 \pm 31 \mathrm{pc}$ for Feige 46 . These agree very well with the distances derived from their Gaia parallaxes, $420_{-14}^{+15} \mathrm{pc}$ and $538_{-19}^{+20} \mathrm{pc}$, respectively, overlapping within $1 \sigma$. In addition, the inferred reddenings, $E(B-V)=0.028 \pm 0.006$ for LS IV $-14^{\circ} 116$ and $0.005 \pm 0.005$ for Feige 46 , are lower than the maximum values of 0.038 and 0.028 along the two lines of sight (Schlafly \& Finkbeiner 2011).

\section{Discussion}

Intermediate He-rich subdwarfs are relatively rare objects (Geier et al. 2017), and some show extreme overabundances of trans-iron elements (Ge, Sr, Y, Zr, and Pb; Jeffery et al. 2017; Wild \& Jeffery 2018). Among these, LS IV-14 116 stands out because its atmosphere is especially enriched in $\mathrm{Sr}, \mathrm{Y}$, and $\mathrm{Zr}$ (Naslim et al. 2013). These high overabundances render these elements detectable not only in their UV spectra, as commonly seen in other sdBs, but also in the optical region. It seems plausible that the atmosphere of Feige 46 may also display similar chemical peculiarities. O'Toole (2004) reported the detection of $\mathrm{Pb}$ in a spectrum of Feige 46 observed with the Goddard High Resolution Spectrograph (GHRS) on board the Hubble Space Telescope (HST; 1320-1520 ̊), but there has been no modern high-resolution optical spectroscopy for this star. Further analysis of the GHRS data, especially in combination with new optical data, would be of utmost interest to address the chemical portrait of Feige 46.

Another peculiar aspect of LS IV $-14^{\circ} 116$ is its membership in the Galactic halo population, which was confirmed by its Galactic velocity components and retrograde orbit around the Galactic center (Randall et al. 2015). We conducted a similar analysis using the Gaia DR2 proper motion and parallax data (Gaia Collaboration 2018). We used the RV value reported by Drilling \& Heber (1987) of $90 \mathrm{~km} \mathrm{~s}^{-1}$ with a more conservative error of $4 \mathrm{~km} \mathrm{~s}^{-1}$. We then followed the procedure described by Randall et al. (2015) to derive the Galactic radial and rotational

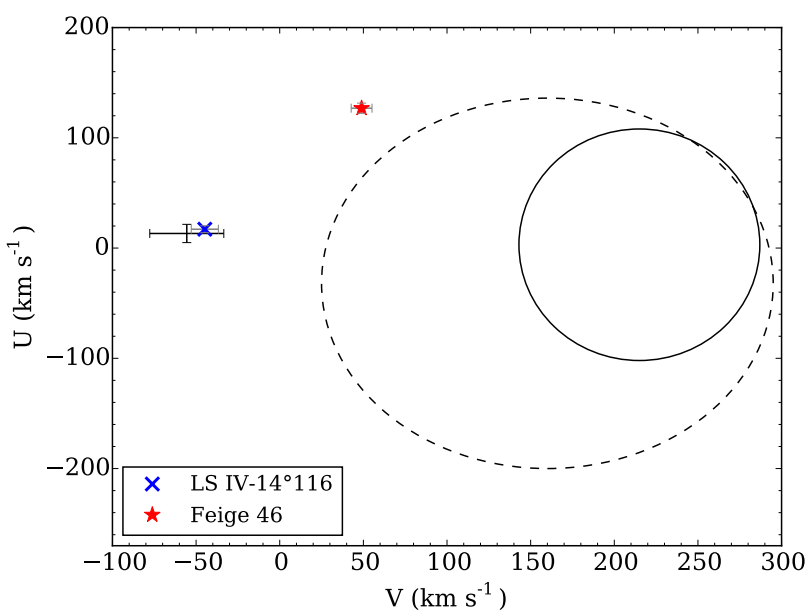

Fig. 3. $U-V$ diagram showing the positions of Feige 46 (star) and LS IV $-14^{\circ} 116$ (cross) compared to the $3 \sigma$ thin-disk (solid line) and thick-disk (dashed line) contours as defined by Pauli et al. (2006). Stars outside these contours have kinematic properties of the halo population. The larger error bars are centered on the values derived by Randall et al. (2015) for LS IV-14 116.

velocity components of Feige $46\left(U=126.9 \pm 4.5 \mathrm{~km} \mathrm{~s}^{-1}, V=\right.$ $\left.48.9 \pm 6.1 \mathrm{~km} \mathrm{~s}^{-1}\right)$, and to recompute those of LS IV $-14^{\circ} 116$ $\left(U=17.1 \pm 3.1 \mathrm{~km} \mathrm{~s}^{-1}, V=-44.8 \pm 7.9 \mathrm{~km} \mathrm{~s}^{-1}\right)$. The positions of the two stars in the $U-V$ diagram are shown in Fig. 3 along with the $3 \sigma$ contours expected for the thin- and thick-disk populations. Like LS IV $-14^{\circ} 116$, Feige 46 lies outside these contours, indicating that it has kinematic properties typical of the halo population. Still, halo kinematics do not necessarily imply that the stars were formed in situ. Some sdBs with extreme kinematics are likely to have acquired such properties after dynamical interactions, for instance, with the central black hole of the Milky Way (Tillich et al. 2011; Ziegerer et al. 2017). Nevertheless, the kinematics of LS IV $-14^{\circ} 116$ and Feige 46 are not particularly extreme, and a halo origin remains a plausible explanation.

The similarity between Feige 46 and LS IV $-14^{\circ} 116$ in terms of both photometric variability and atmospheric properties 
leaves little doubt that the two stars are pulsating through the same mechanism. Their variability cannot be explained by the iron-bump $\kappa$-mechanism that is at work in the vast majority of pulsating hot subdwarfs. Their temperatures and surface gravities place them in the instability region where only $p$-modes would be excited by this mechanism, but no such short-period pulsations are observed in either star, only the unanticipated long-period pulsations. The possibility that a strong magnetic field produces the chemical anomalies and luminosity variability seen in LS IV $-14^{\circ} 116$ was suggested by Naslim et al. (2011), but was later discarded based on spectropolarimetric observations of the star (Randall et al. 2015). The RV variations seen in time-resolved spectroscopy suggest instead that the pulsations are associated with an oscillatory motion of the stellar surface (Jeffery et al. 2015; Martin \& Jeffery 2017).

Currently, the most promising explanation for the variability of these stars is pulsations driven by nuclear burning ( $\epsilon$-mechanism) in helium subflashes following a delayed core He-flash (Miller Bertolami et al. 2011). A delayed core flash occurs after the star has evolved away from the red giant branch, and it can occur as late as when the star is already contracting on the white dwarf cooling curve (Castellani \& Castellani 1993; Lanz et al. 2004). The delayed flash is more explosive and causes additional mixing between the helium-rich material in the core and the hydrogen-rich superficial layers, producing an atmosphere enriched in helium. This late-flasher scenario is therefore an evolutionary channel invoked to explain the formation of helium-enriched hot subdwarfs (Miller Bertolami et al. 2008). In their exploration of the pulsation properties of late-flasher evolutionary models, Battich et al. (2018) showed that long-period $g$-modes can be excited through the $\epsilon$-mechanism in the $\log g-T_{\text {eff }}$ region where both pulsators are found. Although the predicted modes have periods shorter than $2000 \mathrm{~s}$, which is shorter than the observed periods, Battich et al. (2018) pointed out that this could be due to shortcomings in the evolutionary models because the details of this short evolutionary phase are poorly constrained. Interestingly, pulsations excited by the $\epsilon$-mechanism have not yet been confirmed in any type of star (Sowicka et al. 2018). These two He-sdOBVs are currently the most promising candidates for this pulsation mechanism.

An alternative $\kappa$-mechanism was recently suggested by Saio \& Jeffery (2019), in which the excitation is driven by carbon and oxygen opacity bumps. Although their models can excite pulsations in the observed period range, the stellar structure needed to do so, a $0.5 M_{\odot}$ helium main-sequence star with a substantial enrichment of carbon and/or oxygen in the envelope, is very challenging to explain from an evolutionary perspective.

\section{Conclusion}

We have reported the discovery of long-period $g$-mode pulsations in the intermediate He-rich sdOB star Feige 46. The atmospheric parameters and helium abundance of the star confirm that this object belongs to the He-sdOBV pulsating class, which has previously only been defined by the unique prototype LS IV $-14^{\circ} 116$. The excitation mechanism that is responsible for the observed pulsations in both stars is a matter of debate: the two suggested models, the $\epsilon$-mechanism and the $\kappa$-mechanism through carbon and oxygen opacity-bumps, each have their own shortcomings. In this context, the pulsation periods observed in the two stars, and potentially in other similar objects, should be of great help in testing and refining evolutionary models. The kinematic properties of Feige 46 indicate that it belongs to the Galactic halo population, just like LS IV $-14^{\circ} 116$. The halo origin as well as the probable single nature and slow rotation of both stars place further constraints on the evolutionary history of these enigmatic objects.

Acknowledgements. M.L. acknowledges funding from the Deutsche Forschungsgemeinschaft (grant DR 281/35-1). This research has made use of NASA's Astrophysics Data System. We would like to thank Ulrich Heber for useful discussions.

\section{References}

Ahmad, A., \& Jeffery, C. S. 2005, A\&A, 437, L51

Battich, T., Bertolami, M. M. M., Córsico, A. H., \& Althaus, L. G. 2018, A\&A, 614, A136

Bauer, F., \& Husfeld, D. 1995, A\&A, 300, 481

Billères, M., Fontaine, G., Brassard, P., et al. 2000, ApJ, 530, 441

Blanchette, J.-P., Chayer, P., Wesemael, F., et al. 2008, ApJ, 678, 1329

Bloemen, S., Hu, H., Aerts, C., et al. 2014, A\&A, 569, A123

Brassard, P., Fontaine, G., Chayer, P., \& Green, E. M. 2010, in AIP Conf. Ser., eds. K. Werner, \& T. Rauch, 1273, 259

Castellani, M., \& Castellani, V. 1993, ApJ, 407, 649

Charpinet, S., Fontaine, G., Brassard, P., et al. 1997, ApJ, 483, L123

Charpinet, S., Fontaine, G., \& Brassard, P. 2001, PASP, 113, 775

Charpinet, S., Van Grootel, V., Fontaine, G., et al. 2011, A\&A, 530, A3

Charpinet, S., Van Grootel, V., Fontaine, G., et al. 2016, IAU Focus Meeting, 29, 581

Charpinet, S., Giammichele, N., Zong, W., et al. 2018, Open Astron., 27, 112

Drilling, J. S., \& Heber, U. 1987, in IAU Colloq. 95: Second Conference on Faint Blue Stars, eds. A. G. D. Philip, D. S. Hayes, \& J. W. Liebert, 603

Feige, J. 1958, ApJ, 128, 267

Fontaine, G., Brassard, P., Charpinet, S., et al. 2012, A\&A, 539, A12

Fontaine, G., Green, E., Brassard, P., Latour, M., \& Chayer, P. 2014, ASP Conf. Ser., 481,83

Gaia Collaboration (Brown, A. G. A., et al.) 2018, A\&A, 616, A1

Geier, S., Østensen, R. H., Nemeth, P., et al. 2017, Open Astron., 26, 164

Graham, J. A. 1970, PASP, 82, 1305

Green, E. M., Guvenen, B., O’Malley, C. J., et al. 2011, ApJ, 734, 59

Heber, U. 2009, ARA\&A, 47, 211

Heber, U. 2016, PASP, 128, 082001

Hubeny, I., \& Lanz, T. 2011, Astrophysics Source Code Library [record ascl:1109.021]

Jeffery, C. S., Ahmad, A., Naslim, N., \& Kerzendorf, W. 2015, MNRAS, 446, 1889

Jeffery, C. S., Baran, A. S., Behara, N. T., et al. 2017, MNRAS, 465, 3101

Kilkenny, D., Koen, C., O'Donoghue, D., \& Stobie, R. S. 1997, MNRAS, 285, 640

Kilkenny, D., Fontaine, G., Green, E. M., \& Schuh, S. 2010, IBVS, 5927, 1

Kilkenny, D., Worters, H. L., \& Østensen, R. H. 2017, MNRAS, 467, 3963

Kudritzki, R. P. 1976, A\&A, 52, 11

Lanz, T., Brown, T. M., Sweigart, A. V., Hubeny, I., \& Landsman, W. B. 2004, ApJ, 602, 342

Martin, P., \& Jeffery, C. S. 2017, Open Astron., 26, 240

Miller Bertolami, M. M., Althaus, L. G., Unglaub, K., \& Weiss, A. 2008, A\&A, 491, 253

Miller Bertolami, M. M., Córsico, A. H., \& Althaus, L. G. 2011, ApJ, 741, L3

Naslim, N., Jeffery, C. S., Behara, N. T., \& Hibbert, A. 2011, MNRAS, 412, 363

Naslim, N., Jeffery, C. S., Hibbert, A., \& Behara, N. T. 2013, MNRAS, 434, 1920

Østensen, R. H., Degroote, P., Telting, J. H., et al. 2012, ApJ, 753, L17

O'Toole, S. J. 2004, A\&A, 423, L25

Pauli, E.-M., Napiwotzki, R., Heber, U., Altmann, M., \& Odenkirchen, M. 2006, A\&A, 447, 173

Randall, S. K., Bagnulo, S., Ziegerer, E., Geier, S., \& Fontaine, G. 2015, A\&A, 576, A65

Randall, S. K., Calamida, A., Fontaine, G., et al. 2016, A\&A, 589, A1

Saio, H., \& Jeffery, C. S. 2019, MNRAS, 482, 758

Samus, N. N., Kazarovets, E. V., Durlevich, O. V., Kireeva, N. N., \& Pastukhova, E. N. 2017, Astron. Rep., 61, 80

Schlafly, E. F., \& Finkbeiner, D. P. 2011, ApJ, 737, 103

Schuh, S., Huber, J., Dreizler, S., et al. 2006, A\&A, 445, L31

Sowicka, P., Handler, G., \& Jones, D. 2018, MNRAS, 479, 2476

Tillich, A., Heber, U., Geier, S., et al. 2011, A\&A, 527, A137

Van Grootel, V., Charpinet, S., Brassard, P., Fontaine, G., \& Green, E. M. 2013,

A\&A, 553, A97

Wild, J. F., \& Jeffery, C. S. 2018, MNRAS, 473, 4021

Ziegerer, E., Heber, U., Geier, S., et al. 2017, A\&A, 601, A58 


\section{Appendix A: Additional table}

Table A.1. Journal of photometric observations of Feige 46.

\begin{tabular}{lccc}
\hline \hline $\begin{array}{l}\text { Date } \\
(\mathrm{UT})\end{array}$ & $\begin{array}{c}\text { Start of run } \\
(\text { HJD2458170+) }\end{array}$ & $\begin{array}{c}\text { Number of frames } \\
(\mathrm{h})\end{array}$ & Length \\
\hline 2018-Feb-26 & 5.87597 & 425 & 3.613 \\
2018-Feb-27 & 6.72110 & 853 & 7.251 \\
2018-Mar-06 & 13.71209 & 556 & 4.726 \\
2018-Mar-25 & 32.66519 & 300 & 2.550 \\
2018-Mar-27 & 34.76409 & 498 & 4.233 \\
2018-Mar-28 & 35.64764 & 850 & 7.225 \\
2018-Mar-29 & 36.71110 & 286 & 2.431 \\
2018-Apr-02 & 40.61363 & 906 & 7.701 \\
2018-Apr-03 & 41.61282 & 911 & 7.744 \\
2018-Apr-14 & 52.61746 & 771 & 6.554 \\
2018-Apr-15 & 53.61904 & 768 & 6.528 \\
2018-Apr-29 & 67.23060 & 563 & 4.786 \\
2018-Apr-30 & 68.62797 & 271 & 2.304 \\
2018-May-25 & 93.63795 & 441 & 3.749 \\
\hline
\end{tabular}

